Piotr KACPRZYK (Warszawa)

\title{
REGULARITY PROPERTIES OF THE ATTRACTOR TO THE NAVIER-STOKES EQUATIONS
}

Abstract. Existence of a global attractor for the Navier-Stokes equations describing the motion of an incompressible viscous fluid in a cylindrical pipe has been shown already. In this paper we prove the higher regularity of the attractor.

1. Introduction. We consider viscous incompressible fluid motions in a finite cylinder with large inflow and outflow and under boundary slip conditions. The following initial-boundary value problem is examined:

$$
\begin{array}{ll}
v, t+v \cdot \nabla v-\operatorname{div} \mathbb{T}(v, p)=f & \text { in } \Omega^{T}=\Omega \times(0, T), \\
\operatorname{div} v=0 & \text { in } \Omega^{T}, \\
v \cdot \bar{n}=0 & \text { on } S_{1}^{T}=S_{1} \times(0, T), \\
\nu \bar{n} \cdot \mathbb{D}(v) \cdot \bar{\tau}_{\alpha}+\gamma v \cdot \bar{\tau}_{\alpha}=0, \quad \alpha=1,2, & \text { on } S_{1}^{T}, \\
v \cdot \bar{n}=d & \text { on } S_{2}^{T}=S_{2} \times(0, T), \\
\bar{n} \cdot \mathbb{D}(v) \cdot \bar{\tau}_{\alpha}=0, \quad \alpha=1,2, & \text { on } S_{2}^{T}, \\
\left.v\right|_{t=0}=v(0) & \text { in } \Omega, \\
\int_{\Omega} p d x=0, &
\end{array}
$$

where $\Omega \subset \mathbb{R}^{3}, S=S_{1} \cup S_{2}=\partial \Omega, v=v(x, t)=\left(v_{1}(x, t), v_{2}(x, t), v_{3}(x, t)\right) \in \mathbb{R}^{3}$ is the velocity vector of the fluid motion, $p=p(x, t) \in \mathbb{R}^{1}$ the pressure, $f=f(x, t)=\left(f_{1}(x, t), f_{2}(x, t), f_{3}(x, t)\right) \in \mathbb{R}^{3}$ the external force field, $\bar{n}$ the unit outward vector normal to the boundary $S$, and $\bar{\tau}_{\alpha}, \alpha=1,2$, are tangent

2010 Mathematics Subject Classification: 35Q35, 76D03, 76D05.

Key words and phrases: Navier-Stokes equations, inflow-outflow problem, slip boundary conditions, cylindrical domains, global existence of regular solutions. 
vectors to $S . \mathbb{T}(v, p)$ is the stress tensor of the form

$$
\mathbb{T}(v, p)=\nu \mathbb{D}(v)-p I,
$$

where $\nu$ is the constant viscosity coefficient, $I$ the unit matrix and $\mathbb{D}(v)$ is the dilatation tensor

$$
\mathbb{D}(v)=\left\{v_{i, x_{j}}+v_{j, x_{i}}\right\}_{i, j=1,2,3} .
$$

Finally $\gamma>0$ is the slip coefficient.

By $\Omega \subset \mathbb{R}^{3}$ we denote a cylindrical type domain parallel to the $x_{3}$ axis with arbitrary cross section. We assume that $S_{1}$ is the part of the boundary which is parallel to the $x_{3}$-axis and $S_{2}$ is perpendicular to $x_{3}$. Hence

$$
\begin{aligned}
S_{1} & =\left\{x \in \mathbb{R}^{3}: \varphi\left(x_{1}, x_{2}\right)=c_{0},-a<x_{3}<a\right\}, \\
S_{2}(-a) & =\left\{x \in \mathbb{R}^{3}: \varphi\left(x_{1}, x_{2}\right)<c_{0}, x_{3}=-a\right\}, \\
S_{2}(a) & =\left\{x \in \mathbb{R}^{3}: \varphi\left(x_{1}, x_{2}\right)<c_{0}, x_{3}=a\right\},
\end{aligned}
$$

where $a, c_{0}$ are given positive numbers and $\varphi\left(x_{1}, x_{2}\right)=c_{0}$ describes a sufficiently smooth closed curve in the plane $x_{3}=$ const.

To describe the inflow and outflow we define

$$
d_{1}=-\left.v \cdot \bar{n}\right|_{S_{2}(-a)}, \quad d_{2}=\left.v \cdot \bar{n}\right|_{S_{2}(a)},
$$

so $d_{i} \geq 0, i=1,2$, and by $(1.1)_{2,3}$ and (1.2) we have the compatibility condition

$$
\Phi \equiv \int_{S_{2}(-a)} d_{1} d S_{2}=\int_{S_{2}(a)} d_{2} d S_{2}
$$

where $\Phi$ is the flux.

Let us introduce an extension $\alpha=\alpha(x, t) \in \mathbb{R}$ such that

$$
\left.\alpha\right|_{S_{2}(-a)}=d_{1},\left.\quad \alpha\right|_{S_{2}(a)}=d_{2} .
$$

Then equations (1.1) $)_{2,3,6}$ and (1.3) imply the compatibility condition

$$
\int_{\Omega} \alpha_{, x_{3}} d x=-\left.\int_{S_{2}(-a)} \alpha\right|_{x_{3}=-a} d S_{2}+\left.\int_{S_{2}(a)} \alpha\right|_{x_{3}=a} d S_{2}=0 .
$$

In [14, 15, 16] the long time existence of solutions is proved in non-axially cylindrical domains. In [17] the existence is proved in Besov spaces and in [2, 9] the proof of existence is simplified so as to use Sobolev spaces only. In [8] the global existence of solution is proved by prolongation of long time solutions from [9]. In [17] the inflow-outflow problem is considered, and in [3] global existence by prolongation of long time solutions from [2] is proved. 
To formulate the main result of [3] we need the notation

$$
\begin{aligned}
\Gamma^{2}(t)= & |\alpha|_{2, S_{1}}^{2}+\left|\alpha_{, t}\right|_{6 / 5, \Omega}^{2}+\left|\alpha_{, x_{3} t}\right|_{6 / 5, \Omega}^{2} \\
& +\left(1+\|\alpha\|_{1,3, \Omega}^{2}\right)|\nabla \alpha|_{2, \Omega}^{2}+|f|_{6 / 5, \Omega}^{2}, \\
\Gamma_{1}^{2}(k T, t)= & \|\alpha\|_{L_{\infty}\left(k T, t L_{2}(\Omega)\right)}^{2}+\left\|\alpha_{, x_{3}}\right\|_{L_{\infty}\left(k T, t ; L_{2}(\Omega)\right)}^{2}+\int_{k T}^{t}\left\|\alpha\left(t^{\prime}\right)\right\|_{1,2, \Omega}^{2} d t^{\prime}, \\
l_{1}^{2}(k T, t)= & c \exp c\left(\left|d_{1}\right|_{3,6, S_{2} \times(k T, t)}^{6}+|\nabla \alpha|_{3,2, \Omega \times(k T, t)}^{2}\right) \\
& \cdot\left(\int_{k T}^{t} \Gamma^{2}\left(t^{\prime}\right) d t^{\prime}+\Gamma_{1}^{2}(k T, t)+|v(k T)|_{2, \Omega}^{2}\right), \\
G(k T, t)= & l_{1}(k T, t)+\left\|d_{1}\right\|_{3 / 2,2, S_{2} \times(k T, t)}+|f|_{2, \Omega \times(k T, t)} \\
& +\left|F_{3}\right|_{10 / 7, \Omega \times(k T, t)}+\left|d_{1}\right|_{\infty, \Omega \times(k T, t)}, \quad \text { where } \quad F_{3}=(\operatorname{rot} v)_{3}, \\
G^{\prime}(k T, t)= & |g|_{2, \Omega \times(k T, t)}+l_{1}(k T, t)+\left\|d_{1, x^{\prime}}\right\|_{3 / 2,2, S_{2} \times(k T, t),},
\end{aligned}
$$

where $g=f_{, x_{3}}$,

$$
\begin{aligned}
\eta(k T, t)= & \left\|d_{1, x^{\prime}}\right\|_{L_{\infty}\left(k T, t ; H^{1}\left(S_{2}\right)\right)}+\left\|d_{1, t}\right\|_{L_{2}\left(k T, t ; H^{1}\left(S_{2}\right)\right)} \\
& +\left\|f_{3}\right\|_{L_{2}\left(k T, t ; L_{4 / 3}(\Omega)\right)}+\|g\|_{L_{2}\left(k T, t ; L_{6 / 5}(\Omega)\right)}+\frac{1}{T} l_{1}(k T, t),
\end{aligned}
$$

where $t \in(k T,(k+1) T)$.

Theorem 1.1 (global existence). Assume that $t \in(k T,(k+1) T), k \in$ $\mathbb{N} \cup\{0\} \equiv \mathbb{N}_{0}$,

$$
\begin{aligned}
& \int_{k T}^{t} \Gamma^{2}\left(t^{\prime}\right) d t^{\prime} \leq \int_{0}^{T} \Gamma^{2}\left(t^{\prime}\right) d t^{\prime}, \\
& \Gamma_{1}(k T, t) \leq \Gamma_{1}(0, T), \quad G(k T, t) \leq G(0, T), \quad G^{\prime}(k T, t) \leq G^{\prime}(0, T), \\
& l_{1}(k T, t) \leq l_{1}(0, T), \quad \eta(k T, t) \leq \eta(0, T) .
\end{aligned}
$$

Assume that $\eta(k T,(k+1) T)$ is so small that there exists a positive constant A such that

$$
\varphi(A, G(k T, t)) \eta(k T, t)+G^{\prime}(k T, t)<A, \quad t \in(k T,(k+1) T],
$$

where $\varphi$ is some positive increasing function. Then there exists a solution to (1.1) such that

$$
\left\|v_{, x_{3}}\right\|_{W_{2}^{2,1}((k T,(k+1) T) \times \Omega)} \leq A, \quad\|v\|_{W_{2}^{2,1}((k T,(k+1) T) \times \Omega)} \leq c\left(A^{2}+1\right),
$$
where $A>0$ is a constant chosen for a given $T$ and independent of $k \in \mathbb{N}$.

In 4 we proved the existence of the global attractor for problem (1.1). The attractor is bounded in $H^{1}(\Omega)$. In this paper we show the $H^{2}$ regularity for this attractor.

Now we formulate the main result. 
TheOREM 1.2. There exists a global attractor $\mathcal{A}$ in $V$ for the semiprocess $\{S(t)\}_{t \geq 0}$ defined by (3.1). The attractor is bounded in $H^{2}(\Omega)$ and compact and connected in $V$. It attracts bounded sets in $V$, where $V$ is defined by (2.2).

2. Notation and auxiliary results. To simplify the presentation we introduce the following notation:

$$
\begin{array}{ll}
|u|_{p, Q}=\|u\|_{L_{p}(Q)}, & Q \in\left\{\Omega^{T}, S^{T}, \Omega, S\right\}, p \in[1, \infty], \\
\|u\|_{s, Q}=\|u\|_{H^{s}(Q)}, & Q \in\{\Omega, S\}, s \in \mathbb{R}_{+} \cup\{0\}, \\
\|u\|_{s, Q^{T}}=\|u\|_{W_{2}^{s, s / 2}\left(Q^{T}\right)}, & Q \in\{\Omega, S\}, s \in \mathbb{R}_{+} \cup\{0\}, \\
|u|_{p, q, Q^{T}}=\|u\|_{L_{q}\left(0, T ; L_{p}(Q)\right)}, & Q \in\{\Omega, S\}, p, q \in[1, \infty], \\
\|u\|_{s, q, Q^{T}}=\|u\|_{W_{q}^{s, s / 2}\left(Q^{T}\right)}, & Q \in\{\Omega, S\}, s \in \mathbb{R}_{+} \cup\{0\}, q \in[1, \infty], \\
\|u\|_{s, q, Q}=\|u\|_{W_{q}^{s}(Q)}, & Q \in\{\Omega, S\}, s \in \mathbb{R}_{+} \cup\{0\}, q \in[1, \infty] .
\end{array}
$$

By $c$ we denote a generic constant which changes its magnitude from formula to formula. By $\bar{c}(\sigma), \varphi(\sigma)$ we understand generic functions which are always positive and increasing. Finally, we do not distinguish scalar and vector-valued functions in notation.

We introduce the space

$$
\begin{aligned}
& V_{2}^{k}\left(\Omega^{T}\right)=\left\{u:\|u\|_{V_{2}^{k}\left(\Omega^{T}\right)}=\underset{t \in(0, T)}{\operatorname{ess} \sup }\|u\|_{H^{k}(\Omega)}\right. \\
& \left.\quad+\left(\int_{0}^{T}\|\nabla u(t)\|_{H^{k}(\Omega)}^{2} d t\right)^{1 / 2}<\infty\right\}, \quad k \in \mathbb{N} .
\end{aligned}
$$

Finally we introduce the quantities

$$
\begin{aligned}
& h=v_{, x_{3}}, \quad q=p_{, x_{3}}, \quad g=f_{, x_{3}}, \\
& w=v_{3}, \quad \chi=v_{2, x_{1}}-v_{1, x_{2}} .
\end{aligned}
$$

Let $\delta>0$ be fixed and

$$
\bar{V}=\left\{v \in C^{\infty}(\Omega): \operatorname{div} v=0,\left.v \cdot \bar{n}\right|_{S_{1}}=0,\left.v \cdot \bar{n}\right|_{S_{2}}=d,\left|v_{x_{3}}\right|_{2, \Omega}<\delta\right\},
$$

then

$$
\begin{aligned}
& H \equiv \text { closure of } \bar{V} \text { in } L_{2} \text {-norm, } \\
& V \equiv \text { closure of } \bar{V} \text { in } H^{1} \text {-norm. }
\end{aligned}
$$

Lemma 2.1 (Korn inequality; see [14]). Assume that $|\mathbb{D}(v)|_{2, \Omega}^{2}<\infty$, $\left.v \cdot \bar{n}\right|_{S}=0, \operatorname{div} v=0$. If $\Omega$ is not axially symetric, then there exists a constant $c>0$ such that

$$
\|v\|_{1, \Omega}^{2} \leq c|\mathbb{D}(v)|_{2, \Omega}^{2} .
$$

First we need estimates and the uniform Gronwall inequality. 
Lemma 2.2. A solution $v \in H^{2}(\Omega)$ of the elliptic problem

$$
\begin{aligned}
& \operatorname{div} \mathbb{D}(v)=f, \\
& \left.v \cdot \bar{n}\right|_{S_{1}}=0 \\
& \left.v \cdot \bar{n}\right|_{S_{2}}=d, \\
& \left(\bar{n} \cdot \mathbb{D}(v) \cdot \bar{\tau}_{\alpha}+v \cdot \bar{\tau}_{\alpha}\right)_{\left.\right|_{S_{1}}}=0, \quad \alpha=1,2, \\
& \left.\bar{n} \cdot \mathbb{D}(v) \cdot \bar{\tau}_{\alpha}\right|_{S_{2}}=0, \quad \alpha=1,2,
\end{aligned}
$$

satisfies the estimate

$$
\|v\|_{2, \Omega} \leq c\left(|f|_{2, \Omega}+|v|_{2, \Omega}+\|d\|_{3 / 2,2, S_{2}}+\left\|v \cdot \tau_{\alpha}\right\|_{1 / 2,2, S_{1}}\right) .
$$

Lemma 2.3. A solution $(v, p) \in H^{2}(\Omega) \times H^{1}(\Omega)$ of the elliptic problem

$$
\begin{aligned}
& \operatorname{div} \mathbb{T}(v, p)=f, \\
& \operatorname{div} v=0, \\
& \left.v \cdot \bar{n}\right|_{S_{1}}=0, \\
& \left.v \cdot n\right|_{S_{2}}=d, \\
& \left(\bar{n} \cdot \mathbb{D}(v) \cdot \bar{\tau}_{\alpha}+v \cdot \bar{\tau}_{\alpha}\right)_{S_{1}}=0, \quad \alpha=1,2, \\
& \left.\bar{n} \cdot \mathbb{D}(v) \cdot \bar{\tau}_{\alpha}\right|_{S_{2}}=0, \quad \alpha=1,2,
\end{aligned}
$$

satisfies the estimate

$$
\|v\|_{2, \Omega}+|\nabla p|_{2, \Omega} \leq c\left(|f|_{2, \Omega}+|v|_{2, \Omega}+\|d\|_{3 / 2,2, S_{2}}+\left\|v \cdot \tau_{\alpha}\right\|_{1 / 2,2, S_{1}}\right) .
$$

Lemmas 2.2 and 2.3 follow from the general theory on boundary value problems for Douglis-Nirenberg elliptic systems (see [10]).

Lemma 2.4 (uniform Gronwall inequality; see [12, Ch. 3, Lemma 1.1]). Let $g, h, y:\left[t_{0}, \infty\right) \rightarrow(0, \infty)$ be continuous functions. Assume that for some $r>0$ and all $t>t_{0}$ we have

$$
\begin{gathered}
y^{\prime}(t) \leq g(t) y(t)+h(t), \\
\int_{t}^{t+r} g(s) d s \leq a_{1}, \quad \int_{t}^{t+r} h(s) d s \leq a_{2}, \quad \int_{t}^{t+r} y(s) d s \leq a_{3} .
\end{gathered}
$$

Then $y$ satisfies the uniform estimate

$$
y(t+r) \leq\left(a_{3} / r+a_{2}\right) e^{a_{1}} \quad \text { for } t>t_{0} .
$$

We formulate the main results from [4].

TheOREM 2.1. There exists a unique global attractor $\mathcal{A}_{\Sigma}$ in $H$ for the family of semiprocesses $\left\{U_{\sigma}(t, \tau)\right\}_{t \geq \tau \geq 0}, \sigma \in \Sigma$, defined by (3.1). The attractor is bounded in $V$ and compact and connected in $H$. It attracts bounded sets in $H$.

THEOREM 2.2. Let $f$ denote the external field force in the nonstationary problem and $f_{\infty}$ the external field force in the stationary problem. Assume 
that $f_{\infty} \in L_{6 / 5}(\Omega)$. If

$$
\left\|f(t)-f_{\infty}\right\|_{L_{6 / 5(\Omega)}} \underset{t \rightarrow \infty}{\longrightarrow} 0 \quad \text { and } \quad\left\|d-d_{\infty}\right\|_{L_{2}\left(S_{2}\right)} \underset{t \rightarrow \infty}{\longrightarrow} 0,
$$

then the solution $v(t)$ of problem (1.1) converges to the stationary solution $v_{\infty}$ of problem (1.1).

3. Existence of global attractor. In this section we prove the $H^{2}$ regularity of the global attractor to the problem (1.1). We start by recalling some facts and definitions from [1, Ch. 4].

Let us rewrite equation $(1.1)_{1}$ in the abstract form

$$
v_{, t}=A(v, t)=A_{\sigma(t)}(v), \quad t \in \mathbb{R}^{+},
$$

where the right-hand side depends explicitly on the time symbol $\sigma(t)$, which is the collection of all time-dependent coefficients of the equation (in the Navier-Stokes equations that will be the time-dependent external forces). By $\Phi$ we denote some metric or Banach space which contains values of $\sigma(t)$ for a.e. $t \in \mathbb{R}_{+}$. Moreover we assume that $\sigma(t)$, as a function of $t$, belongs to a topological space $\Xi:=\left\{\xi(t), t \in \mathbb{R}_{+}: \xi(t) \in \Psi\right.$ for a.e. $\left.t \in \mathbb{R}_{+}\right\}$.

Replacing the symbol $\sigma(t)$ by the shifted symbol $\sigma(t+h)$ should not change the attractor, hence we introduce a translation invariant subspace $\Sigma \subseteq \Xi$ called the symbol space. Translation invariance means that for all $\sigma(t) \in \Sigma$ the relation $T(h) \sigma(t)=\sigma(t+h) \in \Sigma$ holds, where $T(h): \Xi \rightarrow \Xi$ is the shift operator. In our case, it will be convenient to set $\Sigma=\Sigma\left(\sigma_{0}\right) \equiv$ $\left\{\sigma_{0}(t+h): h \in \mathbb{R}^{+}\right\}$, where $\sigma_{0}$ is the time symbol of the initial equation and the closure is taken in the topology of $\Xi$.

Let $v(t)$ be a weak and global solution of problem (1.1) with initial data $v_{0}=v(0)$. We define the family of semiprocesses $\left\{U_{\sigma}(t, \tau)\right\}_{t \geq \tau \geq 0}$ acting on $H, U(t, \tau): H \rightarrow H$, by the formula

$$
v(t)=U_{\sigma}(t, \tau) v(\tau),
$$

where $v(\tau)$ is the initial condition and $\Sigma \ni \sigma(t)=f(\cdot, t)$ is the external force.

By $\mathcal{B}(H)$ we denote the family of all bounded sets in $H$.

Definition 3.1 (see [1, Ch. 4, Definition 3.2]). A family of processes $\left\{u_{\sigma}(t, \tau)\right\}_{t \geq \tau \geq 0}, \sigma \in \Sigma$, is said to be uniformly bounded if for any $B \in \mathcal{B}(H)$ we have

$$
\bigcup_{\sigma \in \Sigma} \bigcup_{\tau \in \mathbb{R}^{+}} \bigcup_{t \geq \tau} U_{\sigma}(t, \tau) B \in \mathcal{B}(H) .
$$

Definition 3.2 (see [1, Ch. 4, Definition 3.3]). A set $B_{0} \in H$ is said to be uniformly absorbing for the family of processes $\left\{U_{\sigma}(t, \tau)\right\}_{t \geq \tau \geq 0}, \sigma \in \Sigma$, if for any $\tau \in \mathbb{R}^{+}$and for every $B \in \mathcal{B}(H)$ there exists $t_{0}=t_{0}(\tau, B)$ such 
that $\bigcup_{\sigma \in \Sigma} U_{\sigma}(t, \tau) B \subseteq B_{0}$ for all $t \geq t_{0}$. If the set $B_{0}$ is compact, we call the family of processes uniformly compact.

Definition 3.3 (see [1, Ch. 4, Definition 3.4]). A set $P$ belonging to $H$ is said to be uniformly attracting for the family of processes $\left\{U_{\sigma}(t, \tau)\right\}_{t \geq \tau \geq 0}$, $\sigma \in \Sigma$, if for any fixed $\tau \in \mathbb{R}^{+}$,

$$
\lim _{t \rightarrow \infty}\left(\sup _{\sigma \in \Sigma} \operatorname{dist}_{E}\left(U_{\sigma}(t, \tau) B, P\right)\right)=0 .
$$

If the set $P$ is compact, we call the family of processes uniformly asymptotically compact.

Definition 3.4 (see [1, Ch. 4, Definition 3.5]). A closed set $\mathcal{A}_{\Sigma} \subset H$ is said to be the uniform attractor of the family of processes $\left\{U_{\sigma}(t, \tau)\right\}_{t \geq \tau \geq 0}$, $\sigma \in \Sigma$, if it is uniformly attracting set and it is contained in any closed uniformly attracting set $\mathcal{A}^{\prime}$ of $\left\{U_{\sigma}(t, \tau)\right\}_{t \geq \tau \geq 0}, \sigma \in \Sigma$.

The existence of the global attractor follows from the following theorem

Theorem 3.1 (see [1, Ch. 4, Theorem 3.1]). If a family of processes $\left\{U_{\sigma}(t, \tau)\right\}_{t \geq \tau \geq 0}, \sigma \in \Sigma$ is uniformly asymptotically compact, then it has the uniform global attractor $\mathcal{A}_{\Sigma}$. The sef $\mathcal{A}_{\Sigma}$ is compact in $H$.

In [4] we have proved that there exists a bounded, compact and absorbing set $B$ in $H$. Now we will show that this set is in fact compact in $V$. We need to bound this set in $H^{2}(\Omega)$.

Lemma 3.1. Assume that $f_{, t} \in L_{2}\left(k T,(k+1) T ; L_{6 / 5}(\Omega)\right) \cap L_{\infty}(k T$, $\left.(k+1) T ; L_{2}(\Omega)\right)$. Then there exists a bounded and absorbing set in $H^{2}(\Omega)$ for the semiprocess $\{S(t)\}_{t \geq 0}$.

Proof. Let $b=\alpha \bar{e}_{3}$, where $\bar{e}_{3}$ is directed along the $x_{3}$-axis. Let $u$ be defined by

$$
\begin{array}{ll}
\operatorname{div} u=-\operatorname{div} b & \text { in } \Omega, \\
u \cdot \bar{n}=0 & \text { on } S .
\end{array}
$$

Next we define a function $\varphi$ as the solution to the problem

$$
\begin{array}{ll}
\Delta \varphi=-\operatorname{div} b & \text { in } \Omega, \\
\nabla \varphi \cdot \bar{n}=0 & \text { on } S, \\
\int_{\Omega} \varphi d x=0 . &
\end{array}
$$

Therefore, we introduce the new function

$$
w=u-\nabla \varphi=v-(b+\nabla \varphi) \equiv v-\delta,
$$


which is the solution to the problem

$$
\begin{array}{ll}
(w+\delta)_{, t}+(w+\delta) \cdot \nabla(w+\delta)-\operatorname{div} \mathbb{T}(w+\delta, p)=f & \text { in } \Omega, \\
\operatorname{div} w=0 & \text { in } \Omega, \\
w \cdot \bar{n}=0 & \text { on } S_{1}, \\
\nu \bar{n} \cdot \mathbb{D}(w+\delta) \cdot \bar{\tau}_{\alpha}+\gamma(w+\delta) \cdot \bar{\tau}_{\alpha}=0, \quad \alpha=1,2, & \text { on } S_{1}, \\
w \cdot \bar{n}=0 & \text { on } S_{2}, \\
\bar{n} \cdot \mathbb{D}(w+\delta) \cdot \bar{\tau}_{\alpha}=0, \quad \alpha=1,2, & \text { on } S_{2} .
\end{array}
$$

We differentiate $(3.3)_{1}$ with respect to time and take the inner product in $L_{2}(\Omega)$ with $w_{, t}$ to obtain

$$
\begin{aligned}
\frac{1}{2} \frac{d}{d t}\left\|w_{, t}\right\|_{L_{2}(\Omega)}^{2}+\left\|w_{, t}\right\|_{H^{1}(\Omega)}^{2}+\gamma \int_{S_{1}}\left|w_{, t} \cdot \bar{\tau}_{\alpha}\right|^{2} d & \leq \varepsilon\left\|w_{, t}\right\|_{H^{1}(\Omega)}^{2} \\
& +\frac{c}{\varepsilon}\left(\left\|\delta_{, t}\right\|_{H^{1}(\Omega)}^{2}+\left\|f_{, t}\right\|_{L_{6 / 5}(\Omega)}^{2}\right) \\
+\left|\int_{\Omega}\left(w_{, t}+\delta_{, t}\right) \cdot \nabla(w+\delta) w_{, t} d x\right| & +\left|\int_{\Omega}(w+\delta) \cdot \nabla\left(w_{, t}+\delta_{, t}\right) w_{, t} d x\right| .
\end{aligned}
$$

We estimate the last two integrals:

$$
\begin{aligned}
\mid \int_{\Omega}\left(w_{, t}+\delta, t\right) \cdot \nabla & (w+\delta) w_{, t} d x \mid \\
\leq & \int_{\Omega}\left|w_{, t}\right|^{2}(|\nabla w|+|\nabla \delta|) d x+\int_{\Omega}\left|\delta_{, t}\right|\left|w_{, t}\right|(|\nabla w|+|\nabla \delta|) d x \\
\leq & c\left\|w_{, t}\right\|_{L_{2}(\Omega)}^{1 / 2}\left\|w_{, t}\right\|_{H^{1}(\Omega)}^{3 / 2}\left(\|w\|_{H^{1}(\Omega)}+\|\delta\|_{H^{1}(\Omega)}\right) \\
& +\varepsilon\left\|w_{, t}\right\|_{H^{1}(\Omega)}^{2}+c\left\|\delta_{, t}\right\|_{L_{4}(\Omega)}^{2}\left(\|w\|_{H^{1}(\Omega)}^{2}+\|\delta\|_{H^{1}(\Omega)}^{2}\right) \\
\leq & \varepsilon\left\|w_{, t}\right\|_{H^{1}(\Omega)}^{2}+c\left\|w_{, t}\right\|_{L_{2}(\Omega)}^{2}\left(\|w\|_{H^{1}(\Omega)}^{4}+\|\delta\|_{H^{1}(\Omega)}^{4}\right) \\
& +c\left\|\delta_{, t}\right\|_{L_{4}(\Omega)}^{2}\left(\|w\|_{H^{1}(\Omega)}^{2}+\|\delta\|_{H^{1}(\Omega)}^{2}\right)
\end{aligned}
$$

and

$$
\begin{array}{r}
\left|\int_{\Omega}(w+\delta) \cdot \nabla\left(w_{, t}+\delta, t\right) w_{, t} d x\right| \leq\left|\int \delta \cdot \nabla\left(w_{t}+\delta_{t}\right) \cdot w_{t} d x\right|+\left|\int w \cdot \nabla \delta_{t} \cdot w_{t} d x\right| \\
\leq \frac{c}{\varepsilon}\left(\left\|w_{, t}\right\|_{L_{2}(\Omega)}^{2}\|\delta\|_{L_{\infty}(\Omega)}+\|\delta\|_{L_{3}(\Omega)}^{2}\left\|\delta_{, t}\right\|_{H^{1}(\Omega)}^{2}+\|w\|_{H^{1}(\Omega)}^{2}\left\|\delta_{t}\right\|_{H^{1}(\Omega)}^{2}\right) \\
+\varepsilon\left\|w_{, t}\right\|_{H^{1}(\Omega)}^{2} .
\end{array}
$$

Then we obtain

$$
\begin{aligned}
\frac{d}{d t}\left\|w_{, t}\right\|_{L_{2}(\Omega)}^{2} \leq & c\left(\left\|w_{, t}\right\|_{L_{2}(\Omega)}^{2}\left(\|w\|_{H^{1}(\Omega)}^{4}+\|\delta\|_{H^{1}(\Omega)}^{4}+\|\delta\|_{L_{\infty}(\Omega)}\right)\right. \\
& \left.+\left\|\delta_{, t}\right\|_{H^{1}(\Omega)}^{2}\left(\|\delta\|_{H^{1}(\Omega)}^{2}+\|w\|_{H^{1}(\Omega)}^{2}+1\right)+\left\|f_{, t}\right\|_{L_{6 / 5}(\Omega)}^{2}\right) .
\end{aligned}
$$

In view of the uniform Gronwall inequality (Lemma 2.4), we get 


$$
\left\|w_{, t}(t)\right\|_{L_{2}(\Omega)}^{2} \leq\left(b_{3} / T+b_{2}\right) e^{b_{1}}, \quad t \geq T
$$

where

$$
\begin{aligned}
& \int_{k T}^{(k+1) T}\left(\|w(t)\|_{H^{1}(\Omega)}^{4}+\|\delta(t)\|_{H^{1}(\Omega)}^{4}+\|\delta(t)\|_{L_{\infty}(\Omega)}\right) d t \leq b_{1}, \\
& c \int_{k T}^{(k+1) T}\left(\left\|\delta_{, t}(t)\right\|_{H^{1}(\Omega)}^{2}\left(\|\delta(t)\|_{H^{1}(\Omega)}^{2}+\|w(t)\|_{H^{1}(\Omega)}^{2}+1\right)+\left\|f_{, t}(t)\right\|_{L_{6 / 5}(\Omega)}^{2}\right) d t \leq b_{2}, \\
& \int_{k T}^{(k+1) T}\left\|w_{, t}(t)\right\|_{L_{2}(\Omega)}^{2} d t \leq b_{3} .
\end{aligned}
$$

Now we multiply $(1.1)_{1}$ by $\operatorname{div} \mathbb{T}(v, p)$, integrate over $\Omega$ and use the Hölder inequality to get

$$
\begin{aligned}
\|\operatorname{div} \mathbb{T}(v, p)\|_{L_{2}(\Omega)}^{2} \leq & \|v, t\|_{L_{2}(\Omega)}\|\operatorname{div} \mathbb{T}(v, p)\|_{L_{2}(\Omega)} \\
& +\|v\|_{L_{6}(\Omega)}\|\nabla v\|_{L_{3}(\Omega)}\|\operatorname{div} \mathbb{T}(v, p)\|_{L_{2}(\Omega)} \\
& +\|\operatorname{div} \mathbb{T}(v, p)\|_{L_{2}(\Omega)}\|f\|_{L_{2}(\Omega)} .
\end{aligned}
$$

We estimate the second term on the r.h.s. of (3.5).

From Lemma 2.3 we get

$$
\begin{aligned}
\|v\|_{L_{6}(\Omega)} & \|\nabla v\|_{L_{3}(\Omega)}\|\operatorname{div} \mathbb{T}(v, p)\|_{L_{2}(\Omega)} \\
\leq & \nu\|v\|_{L_{6}(\Omega)}\|\nabla v\|_{L_{3}(\Omega)}\left(\|\operatorname{div} \mathbb{D}(v)\|_{L_{2}(\Omega)}+\|\nabla p\|_{L_{2}(\Omega)}\right) \\
\leq & c\|v\|_{L_{6}(\Omega)}\|\nabla v\|_{L_{3}(\Omega)}\left(\|\operatorname{div} \mathbb{D}(v)\|_{L_{2}(\Omega)}\right. \\
& \left.\quad+\|d\|_{3 / 2,2, S_{2}}+\|f\|_{L_{2}(\Omega)}+\|v\|_{L_{2}(\Omega)}+\gamma\left\|v \cdot \bar{\tau}_{\alpha}\right\|_{1 / 2,2, S_{1}}\right) \equiv I .
\end{aligned}
$$

The interpolation inequality, the Young inequality and Lemma 2.2 yield

$$
\begin{aligned}
I \leq & c|v|_{6, \Omega}|\nabla v|_{2, \Omega}^{1 / 2}|\nabla v|_{6, \Omega}^{1 / 2}\left(|\operatorname{div} \mathbb{D}(v)|_{2, \Omega}+\|d\|_{3 / 2,2, S_{2}}\right. \\
& \left.+|f|_{2, \Omega}+|v|_{2, \Omega}+\gamma\left\|v \cdot \bar{\tau}_{\alpha}\right\|_{1,2,2, S_{1}}\right) \\
\leq & c\|v\|_{1, \Omega}^{3 / 2}\|v\|_{2, \Omega}^{1 / 2}\left(|\operatorname{div} \mathbb{D}(v)|_{2, \Omega}+\|d\|_{3,2,2, S_{2}}+|f|_{2, \Omega}+|v|_{2, \Omega}\right. \\
& \left.+\gamma\left\|v \cdot \bar{\tau}_{\alpha}\right\|_{1 / 2,2, S_{1}}\right) \\
\leq & c\|v\|_{1, \Omega}^{3 / 2}\left(|\operatorname{div} \mathbb{D}(v)|_{2, \Omega}^{3 / 2}+\|d\|_{3 / 2,2, S_{2}}^{3 / 2}+|f|_{2, \Omega}^{3 / 2}+|v|_{2, \Omega}^{3 / 2}\right. \\
& \left.+\left(\gamma\left\|v \cdot \bar{\tau}_{\alpha}\right\|_{1 / 2,2, S_{1}}\right)^{3 / 2}\right) \\
\leq & c\left(\varepsilon^{-1}\|v\|_{1, \Omega}^{6}+\varepsilon|\operatorname{div} \mathbb{D}(v)|_{2, \Omega}^{2}\right. \\
& \left.+\varepsilon|f|_{2, \Omega}^{2}+\varepsilon|v|_{2, \Omega}^{2}+\varepsilon\|d\|_{3 / 2,2, S_{2}}^{2}+\varepsilon \gamma^{2}\left\|v \cdot \bar{\tau}_{\alpha}\right\|_{1 / 2,2, S_{1}}^{2}\right) .
\end{aligned}
$$


Then from (3.5) we obtain

$$
\begin{aligned}
\|\operatorname{div} \mathbb{T}(v, p)\|_{L_{2}(\Omega)}^{2} \leq & c\left(\left\|v_{, t}\right\|_{L_{2}(\Omega)}^{2}+\|v\|_{H^{1}(\Omega)}^{6}+\varepsilon\|\operatorname{div} \mathbb{D}(v)\|_{L_{2}(\Omega)}^{2}\right. \\
& \left.+\|f\|_{L_{2}(\Omega)}^{2}+\|d\|_{3 / 2,2, S_{2}}^{2}\right)
\end{aligned}
$$

where the first two norms on the r.h.s. are estimated by (2.8).

Finally from (2.5), (3.5) and (3.6) we conclude that

$$
\|v(t)\|_{H^{2}(\Omega)}<\infty \quad \text { for almost all } t>T \text {. }
$$

Hence there exists a ball $\mathcal{B}\left(0, \rho_{3}\right) \subset H^{2}(\Omega)$ centered at 0 with radius $\rho_{3}$ sufficiently large so that $v(t) \in \mathcal{B}\left(0, \rho_{3}\right)$ for almost all $t>t_{0}=t_{0}\left(v_{0}\right)$.

In view of Theorem 2.1, there exists a global attractor in $V$, namely we have proved the following

THEOREM 3.2. There exists a global attractor $\mathcal{A}$ in $V$ for the semiprocess $\{S(t)\}_{t \geq 0}$ defined by (3.1). The attractor is bounded in $H^{2}(\Omega)$ and compact and connected in $V$. It attracts bounded sets in $V$.

Acknowledgments. The author thanks Prof. W. Zajączkowski for important discussions during the preparation of this paper.

This research was partially supported by the Polish KBN Grant 1/P03A $/ 021 / 30$.

\section{References}

[1] V. Chepyzhov and M. Vishik, Attractors for Equations of Mathematical Physics, Amer. Math. Soc., Providence, RI, 2001.

[2] P. Kacprzyk, Global regular nonstationary flow for the Navier-Stokes equations in a cylindrical pipe, Appl. Math. (Warsaw) 34 (2007), 289-307.

[3] -, Global existence for the inflow-outflow problem for the Navier-Stokes equations in a cylinder, ibid. 36 (2009), 195-212.

[4] -, Global attractor for the Navier-Stokes equations in a cylindrical pipe, Ann. Polon. Math. 97 (2010), 201-217.

[5] O. A. Ladyzhenskaya and V. A. Solonnikov, On some problems of vector analysis and generalized boundary value problems for the Navier-Stokes equations, Zap. Nauchn. Sem. LOMI 59 (1976), 81-116 (in Russian).

[6] O. A. Ladyzhenskaya, V. A. Solonnikov and N. N. Ural'tseva, Linear and Quasilinear Equations of Parabolic Type, Nauka, Moscow, 1967 (in Russian).

[7] B. Nowakowski and W. M. Zajączkowski, Global attractor for Navier-Stokes equations in cylindrical domains, Appl. Math. (Warsaw) 36 (2009), 183-194.

[8] -, - Global existence of solutions to Navier-Stokes equations in cylindrical domains, ibid. 36 (2009), 169-182.

[9] J. Rencławowicz and W. M. Zajacczkowski, Large time regular solutions to NavierStokes equations in cylindrical domains, Topol. Methods Nonlinear Anal. 32 (2008), 69-87.

[10] V. A. Solonnikov, On general boundary value problems for Douglis-Nirenberg elliptic systems, Trudy Mat. Inst. Steklov. 92 (1966), 233-297 (in Russian). 
[11] R. Temam, Navier-Stokes Equations, North-Holland, 1979.

[12] - Infinite-Dimensional Dynamical Systems in Mechanics and Physics, Appl. Math. Sci. 68, Springer, 1997.

[13] M. Wiegner and W. M. Zajacczkowski, On stability of axially symmetric solutions to Navier-Stokes equations in a cylindrical domain and with boundary slip conditions, in: Regularity and Other Aspects of the Navier-Stokes Equations, Banach Center Publ. 70, Inst. Math., Polish Acad. Sci., 2005, 251-278.

[14] W. M. Zajączkowski, Long time existence of regular solutions to Navier-Stokes equations in cylindrical domains under boundary slip conditions, Studia Math. 169 (2005), 243-285.

[15] -, Global existence of axially symmetric solutions to Navier-Stokes equations with large angular component of velocity, Colloq. Math. 100 (2004), 243-263.

[16] - Global special regular solutions to the Navier-Stokes equations a cylindrical domain without the axis of symmetry, Topol. Methods Nonlinear Anal. 24 (2004), 60-105.

[17] - Global regular nonstationary flow for the Navier-Stokes equations in a cylindrical pipe, ibid. 26 (2005), 221-286.

Piotr Kacprzyk

Institute of Mathematics and Cryptology

Cybernetics Faculty

Military University of Technology

Kaliskiego 2

00-908 Warszawa, Poland

E-mail: pk_wat@wp.pl

Received on 28.10.2009;

revised version on 30.4.2010 
